\title{
Improved Loop Execution Modeling in the Clang Static Analyzer*
}

\author{
Péter Szécsi ${ }^{a b}$, Gábor Horváth ${ }^{a c}$, and Zoltán Porkoláb ${ }^{a d}$
}

\begin{abstract}
The LLVM Clang Static Analyzer is a source code analysis tool which aims to find bugs in $\mathrm{C}, \mathrm{C}++$, and Objective-C programs using symbolic execution, i.e. it simulates the possible execution paths of the code. Currently the simulation of the loops is somewhat naive (but efficient), unrolling the loops a predefined constant number of times. However, this approach can result in a loss of coverage in various cases.

This study aims to introduce two alternative approaches which can extend the current method and can be applied simultaneously: (1) determining loops worth to fully unroll with applied heuristics, and (2) using a widening mechanism to simulate an arbitrary number of iteration steps. These methods were evaluated on numerous open source projects, and proved to increase coverage in most of the cases. This work also laid the infrastructure for future loop modeling improvements.
\end{abstract}

Keywords: static analysis, symbolic execution, loop modeling

\section{Introduction}

During software development it is natural to make mistakes. Consequently, writing various test cases is required in order to validate the behavior of the program. In addition to the costs of test writing, it is possible that the developers fail to cover all possible critical cases. Furthermore, test writing and running often happens later than code development, but the costs of error correction increases proportionally to elapsed time [2]. This proves that testing alone is not necessarily sufficient to ensure code quality.

The static analysis tools offer a different approach for code validation $[6,1]$. Moreover, they can potentially check for some characteristics of the code - which

* This study was supported by the ÚNKP-17-2 New National Excellence Program of the Hungarian Ministry of Human Capacities and by the EFOP-3.6.2-16-2017-00013

${ }^{a}$ Department of Programming Languages and Compilers, Eötvös Loránd University, Budapest, Hungary

${ }^{b}$ E-mail: ps95@caesar.elte.hu, ORCID: 0000-0001-9156-1337

${ }^{c}$ E-mail: xazax@caesar.elte.hu, ORCID: 0000-0002-0834-0996

${ }^{d}$ E-mail: gsd@caesar.elte.hu, ORCID: 0000-0001-6819-0224 
cannot be verified by testing - e.g. the adherence to conventions. Unfortunately, it is impossible to detect every bug using static analysis [8] without a large number of spurious warnings. Static analyzer tools might not be able to discover some bugs (these are called false negatives) or report correct code snippets as incorrect (false positives). As the developer's time is one of the most valuable resource in the industry and reports of the automated tools are evaluated manually, industrial tools aim to keep the ratio of the false positive reports low while still be able to find real bugs.

The purpose of the Clang Static Analyzer is to find bugs by performing a symbolic execution $[4,3]$ on the code. During symbolic execution, the program is being interpreted, on a function-by-function basis, without any knowledge about the runtime environment. It builds up and traverses an inner model of the execution paths, called ExplodedGraph, for each analyzed function.

The Static Analyzer - as it is indicated by its name - build around the Clang compiler [5]. An important technical note is that the building of the ExplodedGraph is based on the Control Flow Graph (CFG) of the functions. The CFG represents a source-level, intra-procedural control flow of a statement. This statement can potentially be an entire function body, or just a single expression. The CFG consists of CFGBlocks which are simply containers of statements. The CFGBlockss essentially represent the basic blocks of the code but can contain some extra custom information. Although basic blocks and CFGBlocks are technically different, in the rest of the article the term basic blocks will be used for CFGBlocks as well for the sake of easier understanding and better illustration.

Thus during the analysis - based on the function CFGs - an ExplodedGraph is built up. A node of this graph (called ExplodedNode) contains a ProgramPoint (which determines the location) and a State (which contains any known information at that point). Its paths from the root to the leaves are modeling the different execution paths of the analyzed function. Whenever the execution encounters a branch, a corresponding branch will be created in the ExplodedGraph during the simulated interpretation. Hence, branches lead to an exponential number of ExplodedNodes. This combinatorial explosion is handled in the Static Analyzer by stopping the analysis when given conditions are fulfilled. Terminating the analysis process may cause loss of potential true positive results, but it is indispensable for maintaining a reasonable resource consumption regarding the memory and CPU usage.

These conditions are modeled by the concept of budget. The budget is a collection of constraints on the shape of the ExplodedGraph including:

1. The maximum number of traversed nodes in the ExplodedGraph. If this number is reached then the analysis of the simulated function stops.

2. The size of the simulated call stack. When a function call is reached then the analysis continues in its body as if it was inlined to the place of call (interprocedural). There are several heuristics that may control the behavior of inlining process. For example the too large functions are not inlined at all, and the really short functions are not counted in the size of call stack. 
3. The number of times a function is inlined. The idea behind this constraint is that the more a function is analyzed, the less likely it is that a bug will appear in it. If this number is reached then that function will not be inlined again in this ExplodedGraph.

4. The number of times a basic block is processed during the analysis. This constraint limits the number of loop iterations. When this threshold is reached the currently analyzed execution path is aborted. The budget expression can be used in two ways. Sometimes it means the collection of the limitations above, sometimes it refers to one of these limitations. This will always be distinguishable from the context.

\section{Motivation}

Currently, the analyzer handles loops quite simply. It unrolls them 4 times by default and then cuts the analysis of the path where the loop would have been unrolled more than 4 times. This behavior is enforced by the above presented basic block visiting budget.

One of the problems with this approach to loop modeling is the loss of coverage. Specifically, in cases where the loop is statically known to make more than 4 steps, the analyzer do not analyze the code following the loop. Thus, the naive loop handling (described above) could lead to entirely unchecked code. Listing 1 shows a small example exercising this behavior.

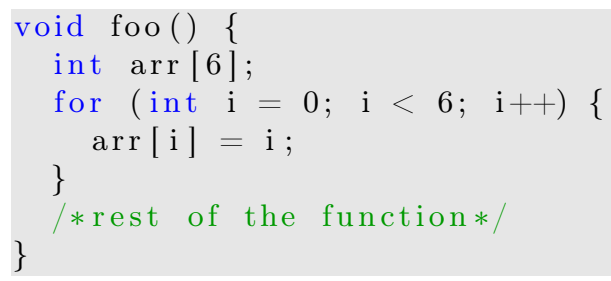

Listing 1: Since the loop condition is known at every iteration, the analyzer will not check the 'rest of the function' part in the current state.

According to the budget rule concerning the basic block visit count, the analysis of the loop stops in the fourth iteration even if the loop condition is simple enough to see that unrolling the whole loop would not be too much extra work relatively. Running out of the budget implies (in this case) that the rest of the function body remains unanalyzed, which may lead to not finding potential bugs. Another problem can be seen on Listing 2 : 


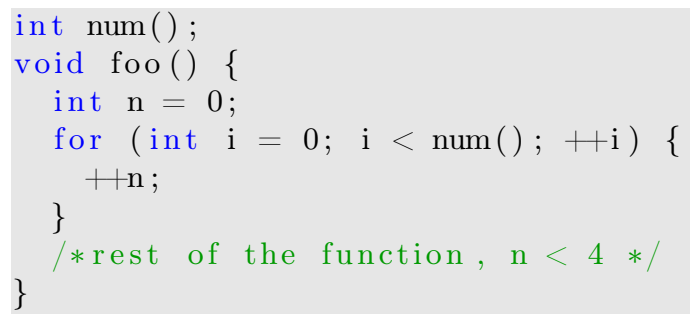

Listing 2: The loop condition is unknown but the analyzer will not generate a simulation path where $\mathrm{n} \geq 4$ (which can result coverage loss).

This code fragment results in an analysis which keeps track of the values of $\mathrm{n}$ and $i$ variables (this information is stored in the State). In every iteration of the loop the values are updated accordingly. Note that updating the State means that a new node is inserted into the ExplodedGraph with the new values. Since the body of the num() function is unknown, the analyzer can not find out its return value. Thus it is considered as unknown. This circumstance makes the graph to split into two branches. The first one belongs to the symbolic execution of the loop body assuming that the loop condition is true. The other one simulates the case where the condition is false and the execution continues after the loop. This process is done for every loop iteration, however, at the 4th time, assuming the condition is true, the path will be cut according to the budget rule. Even though the analyzer generates paths to simulate the code after the loop in the above described case, the value of variable $\mathrm{n}$ will always be less than 4 on these paths and the rest of the function will only be checked with this assumption. This can result in coverage loss as well, since the analyzer will ignore the paths where $\mathrm{n}$ is more than 4 .

\section{Proposed Solution}

In this section two solutions are presented to resolve the above mentioned limitations on symbolic execution of loops in the Clang Static Analyzer. It is important to note that these enhancements are incremental in the sense that the analyzer falls back to the original method on examples which are too complex to handle at the moment. For the sake of simplicity we will use a "division by zero" bug to illustrate the analyzer's behavior in the following examples.

\subsection{Loop Unrolling Heuristics}

We have identified heuristics and patterns (such as loops with small number of branches and small known static bound) in order to find specific loops which are worth to be completely unrolled. This idea is inspired by the following example:

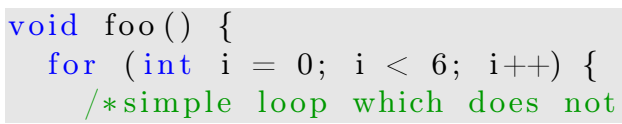




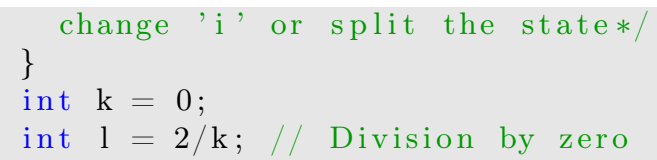

Listing 3: Complete unrolling of the loop makes it possible to find the division by zero error.

Currently, a loop has to fulfill the following conditions in order to be unrolled:

1. The loop condition should arithmetically compare a variable - which is known at the beginning of the loop - to a literal (like: $i<6$ or $6 \geq i$ )

2. The loop should modify the loop variable only once per iteration in its body and the difference needs to be constant. (This way the maximum number of steps can be estimated.)

3. There is no alias created to the loop variable.

4. The estimated number of steps should be less than 128. (Simulating loops which takes thousands of steps because they could single handedly exhaust the budget.)

5. The loop must not generate new branches or use goto statements.

By using this method, the bug on the Listing 3 example is found successfully.

\subsection{Loop Widening}

The final aim of widening is quite the same as the unrolling, to increase the coverage of the analysis. However, it achieves its goal in a very different way. During widening the analyzer simulates the execution of an arbitrary number of iterations. The analyzer already had a widening algorithm which reaches this behavior by discarding all of the known information before the last step of the loop. So the analyzer creates the paths for the first 3 steps and simulate them as usual, but in order to avoid losing the first precise simulation branches, the widening (i.e. the invalidating) happens before the 4th step. This way the coverage will be increased, however, this method is disabled by default, since it can easily result in too much false positives. Consider the example on Listing 4.

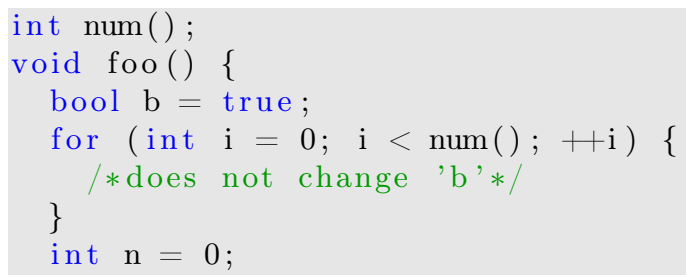




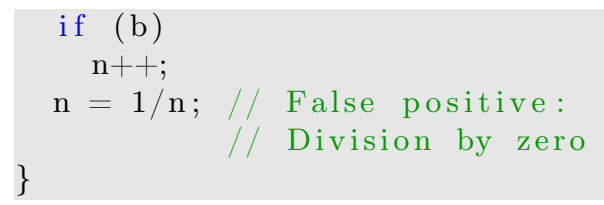

Listing 4: Invalidating every known information (even those which are not modified by the loop) can easily result in false positives.

In this case the analyzer will check that unfeasible path where the variable $\mathrm{b}$ is false, so $\mathrm{n}$ is not incremented and lead into a division by zero error. Since this execution path would never be performed while running the analyzed program, it is considered a false positive. Our aim was to give a more precise approach for widening. There was already conversation within the community about some possible enhancements [7].

One of the main principles is that the analysis should still continue after the block visiting budget is exhausted and the information of only those variables should be invalidated which are possibly modified by the loop, e.g. a statement, like arr [i] $=i$ where $i$ is the loop variable, means that we discard the data on the whole arr array but nothing else. For this reason we developed a solution which checks every possible way in which a variable can be modified in the loop. Then these cases are evaluated and if it encounters a modified variable which cannot be handled by the invalidation process (e.g.: a pointer variable), then the loop will not be widened and we return to the conservative method. This mechanism ensures that we do not create nodes that contain invalid states. This approach helps us to cover cases and find bugs like the one illustrated on Listing 5 without reporting false positives presented on Listing 4.

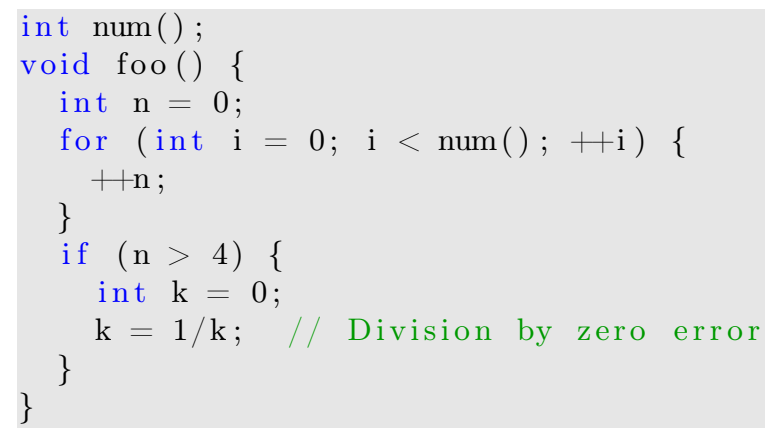

Listing 5: Invalidating the information on only the possible changed variables can result higher coverage (while limiting the number of the found false positives).

The bug is found by invalidating the known information on variable $\mathrm{n}$ (and $\mathrm{i}$ as well). This makes the analyzer to create a branch where it checks the body of the if statement and finds the bug. However, this solution has its own limitations when dealing with nested loops. Consider the case on Listing 6 . 


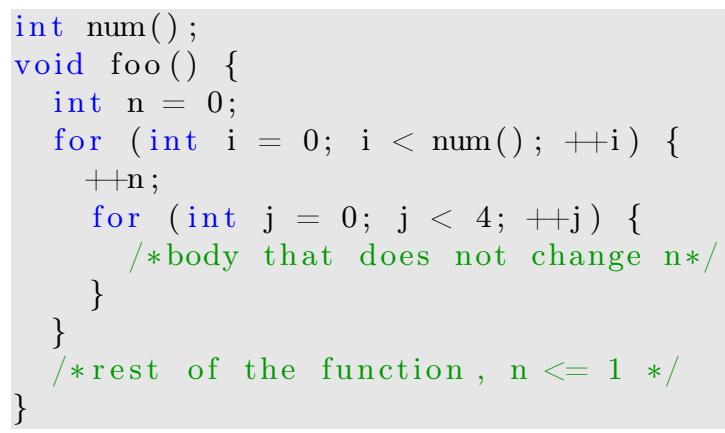

Listing 6: The naive widening method does not handle well the nested loops. In this example the outer loop will not be widened.

In this scenario, when the analyzer first step into the outer loop (so it assumes that $i<$ num() is true) and encounter the inner loop, it consumes its (own) block visiting budget. (This implies that it will be widened, although in this case it means that only the inner loop counter $(\mathrm{j})$ information is discarded.) After moving on to the next iteration, we may assume that we are on the path where the outer loop condition is true again. Due to the fact that the budget was already exhausted in the previous iteration, the next visit of the first basic block of the inner loop (the condition) means that this path will be completely cut off and not analyzed. This results in the outer loop not reaching the step number where it would been widened. Furthermore, the outer loop will not even reach the 3rd step, even the 2nd is stopped at in its body (as described above). This causes the problem that even though the loop widening method is used, the rest of the function will be analyzed by the assumption $\mathrm{n}<=1$.

In order to deal with the above described nested loop problem, we have implemented a replay mechanism. This means that whenever we encounter an inner loop which already consumed its budget, we replay the analysis process of the current step of the outer loop after performing a widening first. This ensures the creation of a path which assumes that the condition is false and simulates the execution after the loop while the possibly changed information are discarded. This way the analyzer will not exclude some feasible path because of the simple loop handling which solves the problem.

An additional note to the widening process is that it makes sense to analyze the branch where the condition is true with the widened State as well. The example on Listing 7 shows a case where this is useful.

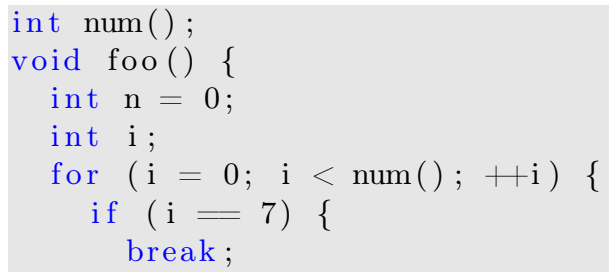




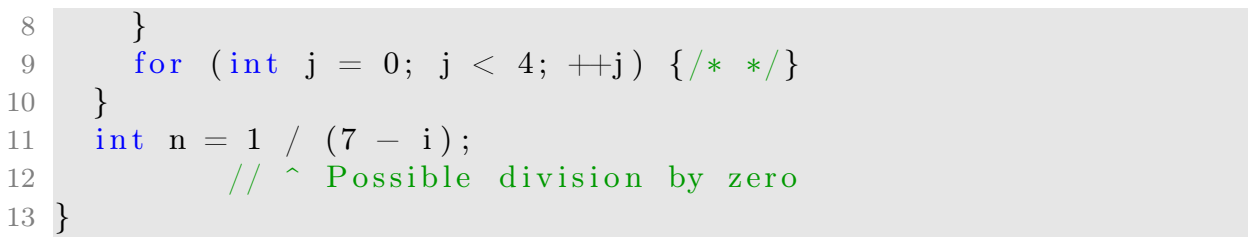

Listing 7: The replay mechanism successfully helps us to find the possible error the outer loop.

This way the analyzer will produce a path where the value of $i$ is known to be 7 , so it will be able find the possible division by zero error.

\section{Evaluation}

The effect of the described loop modeling approaches was measured on various popular $\mathrm{C} / \mathrm{C}++$ open source projects. These are the following:

\begin{tabular}{|c||c|c|}
\hline Project & LoC & Language \\
\hline TinyXML & $20 \mathrm{k}$ & $\mathrm{C}++$ \\
\hline Curl & $21 \mathrm{k}$ & $\mathrm{C}$ \\
\hline Redis & $40 \mathrm{k}$ & $\mathrm{C}$ \\
\hline Xerces & $228 \mathrm{k}$ & $\mathrm{C}++$ \\
\hline Vim & $540 \mathrm{k}$ & $\mathrm{C}$ \\
\hline OpenSSL & $550 \mathrm{k}$ & $\mathrm{C}$ \\
\hline PostgreSQL & $950 \mathrm{k}$ & $\mathrm{C}$ \\
\hline FFmpeg & $1080 \mathrm{k}$ & $\mathrm{C}$ \\
\hline
\end{tabular}

\subsection{Coverage and the number of explored paths}

Keeping track of these statisics are already part of the analyzer. The coverage percentage is based on the ratio of the visited and the total number of basic blocks in the analyzed functions (instead of the number of visited statements), which results in a small imprecision. It is important to note that the introduced loop modeling methods require having additional loop entrance and exit point information in the CFG. This can lead to having more basic blocks in the CFG and it can affect the statistics. As a result, even statistics produced by using the current loop modeling approach were measured with this information added to the CFG.

The coverage and the number of explored paths are generated for every translation unit and then summarized. This means that header files which are included in more than one translation unit can influence more statistics. However, by using this summarization process consistently for every measurement the results reflect the reality. 
The tables presented in this section summarize measurement results using different loop modeling approaches: the current practice (denoted by Normal) and the hereby introduced loop unrolling (Unroll) and loop widening (Widen) methods separately and simultaneously $(\mathrm{U}+\mathrm{W})$.

Table 1 shows the coverage difference using the introduced approaches. On most of the projects, analysis coverage was strictly increased by using any of the proposed approaches. The widening method had a stronger influence on the coverage in the average case. However, the complete unroll of specific loops could result in a higher coverage as well (e.g. Curl, Redis). In general, enabling both of them was the most beneficial with respect to the coverage.

Table 2 presents the numbers of analyzed execution paths. As expected, both introduced loop modeling methods resulted in a higher number of simulated paths on (almost) all of the projects. The only exception is the unrolling approach on the FFmpeg project, which caused the budget limiting the number of traversed

Table 1: The code coverage of the analysis on the evaluated projects expressed in percentage

\begin{tabular}{|c||c|c|c|c|}
\hline Project & Normal & Unroll & Widen & $\mathrm{U}+\mathrm{W}$ \\
\hline \hline TinyXML & 84.2 & 84.2 & 85.1 & 85.1 \\
\hline Curl & 76.2 & 76.9 & 77.7 & 77.2 \\
\hline Redis & 68.5 & 69.1 & 68.5 & 71.3 \\
\hline Xerces & 92.3 & 92.4 & 92.7 & 92.7 \\
\hline Vim & 60.4 & 60.6 & 60.6 & 60.7 \\
\hline OpenSSL & 97.4 & 97.5 & 97.7 & 97.7 \\
\hline PostgreSQL & 76.9 & 77.0 & 76.9 & 76.9 \\
\hline FFmpeg & 86.1 & 86.3 & 87.0 & 86.8 \\
\hline
\end{tabular}

Table 2: The numbers of explored execution paths using different loop modeling approaches

\begin{tabular}{|c||r|r|r|r|}
\hline Project & Normal & Unrolling & Widening & $\mathrm{U}+\mathrm{W}$ \\
\hline \hline TinyXML & 14452 & 15460 & 14765 & 15773 \\
\hline Curl & 18272 & 18577 & 28835 & 24279 \\
\hline Redis & 69857 & 70097 & 98446 & 100929 \\
\hline Xerces & 395615 & 398077 & 430989 & 433358 \\
\hline Vim & 155451 & 157266 & 188136 & 173121 \\
\hline OpenSSL & 687175 & 687932 & 700464 & 701013 \\
\hline PostgreSQL & 382660 & 383874 & 453188 & 419118 \\
\hline FFmpeg & 466613 & 458480 & 571399 & 521725 \\
\hline
\end{tabular}


ExplodedNodes to exhaust earlier, slightly decreasing the number of checked paths. Enabling both of the features resulted in similar or fewer number of explored paths than the runs using only widening. This effect can be explained in two ways: (1) the analyzer prefers to completely unroll loops rather than widen them, which results in a more precise modeling of the state and can exclude unfeasible paths, (2) the simultaneous use of the methods can lead to exhausting the budget on earlier paths, where the analysis will be terminated.

\subsection{Found bugs}

The number of bug reports using the different loop modeling methods can be seen in Table 3. The increase in analysis coverage and in the number of checked paths usually implies an increased number of found bugs, which indeed can be observed on the numbers. However, it is important to note that the upsurge of the number of explored execution paths described in Table 2 considerably outweighs the moderate rise in the number of bug reports. In some cases enabling a proposed feature could result in less results due to two important factors: (1) the more information we collect by precisely analyzing the execution paths does not result in false conclusion, (2) the global budget is exceeded for exploring new paths and some of the earlier checked will be skipped. Unfortunately, case (2) is a possible scenario, however, the increased coverage using the described features shows that this way we still explore more interesting cases. Since the loop widening method creates more new paths by discarding information on the values of variables, it could introduce the risk of analyzing paths that lead to false positives. However, from the results it seems that this was not a problem in practice: relative to the increase in the number of analyzed paths, the number of reports hardly increased. Moreover, based on studying the environment of the found bugs, the ratio of false positive findings was low (beside some clear true positive) among the newly detected bugs.

Table 3: The number of bug reports produced by the analyzer.

\begin{tabular}{|c||c|c|c|c|}
\hline Project & Normal & Unrolling & Widening & $\mathrm{U}+\mathrm{W}$ \\
\hline \hline TinyXML & 1 & 1 & 3 & $3(+200 \%)$ \\
\hline Curl & 16 & 16 & 16 & $16(0 \%)$ \\
\hline Redis & 55 & 58 & 55 & $59(+7.27 \%)$ \\
\hline Xerces & 62 & 62 & 61 & $61(-1.61 \%)$ \\
\hline Vim & 74 & 74 & 76 & $78(+5.4 \%)$ \\
\hline OpenSSL & 152 & 152 & 153 & $153(+0.66 \%)$ \\
\hline PostgreSQL & 323 & 323 & 327 & $331(+2.48 \%)$ \\
\hline FFmpeg & 425 & 420 & 423 & $454(+6.82 \%)$ \\
\hline
\end{tabular}




\subsection{Analysis time}

The running time on different projects is showed in Table 4. Although the widening method lead into more analyzed execution paths, the analysis time increase was more intense after enabling the unrolling process. This is possible due to the fact that unrolling leads to long paths where the State usually contains more information (constraints on variable values), which is very expensive in respect of running time. In general there was a manageable increase in the analysis time at all examined projects which suggests a good scalability of the proposed improvements.

Table 4: Average measured time of the analysis expressed in minutes. (Average of 5 runs.)

\begin{tabular}{|c||c|c|c|c|}
\hline Project & Normal & Unrolling & Widening & $\mathrm{U}+\mathrm{W}$ \\
\hline \hline TinyXML & $0: 51$ & $0: 51$ & $0: 52$ & $0: 52(+2 \%)$ \\
\hline Curl & $0: 50$ & $1: 06$ & $0: 55$ & $1: 05(+30 \%)$ \\
\hline Redis & $2: 06$ & $2: 11$ & $2: 28$ & $2: 10(+3 \%)$ \\
\hline Xerces & $3: 38$ & $3: 34$ & $3: 37$ & $3: 39(+0.5 \%)$ \\
\hline Vim & $3: 11$ & $3: 26$ & $3: 18$ & $3: 27(+3 \%)$ \\
\hline OpenSSL & $2: 04$ & $2: 22$ & $2: 13$ & $2: 19(+8.3 \%)$ \\
\hline PostgreSQL & $7: 03$ & $8: 32$ & $7: 48$ & $7: 59(+13 \%)$ \\
\hline FFmpeg & $9: 40$ & $10: 22$ & $10: 14$ & $11: 20(+17 \%)$ \\
\hline
\end{tabular}

\section{$5 \quad$ Future work}

The heuristic patterns for completely unrolled loops could be extended to involve loops whose bound is a known variable which is not changed in the body. Furthermore, even more general rules would be beneficial: consider loops where the value variables are known at the beginning and they are affected by a known constant change by every iteration. These improvements have not been implemented yet due to some technical and framework limitations.

During the widening process we invalidate any possibly changed information. However, a change made on a pointer could mean that we need to invalidate all variables due to the lack of advanced pointer analysis. Therefore, introducing pointer analysis algorithms to the analyzer could help to develop a more precise invalidation process.

The infrastructural improvements enable the analyzer to provide entry points for bug finding modules (checkers) on loop entrances/exits and identify the currently simulated loop for every ExplodedNode. On top of these entry points new checkers can be implemented. 


\section{Conclusion}

Two alternative approaches was introduced for improving the simulation of loops during symbolic execution. These were implemented and subsequently evaluated on various open source projects, with a clear improvement of code coverage in general. The new methods make it possible to explore previously skipped, feasible execution paths, especially when both of them are used in conjunction.

The required changes done to the underlying infrastructure should also ease the implementation of future enhancements. In particular, information tracked by the analysis about location contexts were expanded with additional fields. While code coverage was measured to have increased by an average of $0.8 \%$ and the number of explored execution paths by an average of $16 \%$, there was a noticeable performance penalty as well. A general increase in the execution time was observed, with an average of $9.5 \%$. The number of simulated paths also increased proportionally with the time taken, suggesting this time was well spent. In conclusion, if the user does not mind taking $\sim 10 \%$ more time for a more comprehensive analysis, then it is beneficial to enable the proposed feature set by default.

\section{Acknowledgment}

We would like to thank to the members of the CodeChecker team at Ericsson for their valuable and helpful suggestions on the paper.

\section{References}

[1] Bessey, Al, Block, Ken, Chelf, Ben, Chou, Andy, Fulton, Bryan, Hallem, Seth, Henri-Gros, Charles, Kamsky, Asya, McPeak, Scott, and Engler, Dawson. A few billion lines of code later: Using static analysis to find bugs in the real world. Commun. ACM, 53(2):66-75, February 2010. DOI: 10.1145/1646353. 1646374 .

[2] Boehm, Barry and Basili, Victor R. Software defect reduction top 10 list. Computer, 34(1):135-137, January 2001. DOI: 10.1109/2.962984.

[3] Hampapuram, Hari, Yang, Yue, and Das, Manuvir. Symbolic path simulation in path-sensitive dataflow analysis. SIGSOFT Softw. Eng. Notes, 31(1):52-58, September 2005. DOI: 10.1145/1108768.1108808.

[4] King, James C. A new approach to program testing. In Proceedings of the international conference on Reliable software, 1975.

[5] Lattner, Chris. LLVM and Clang: Next generation compiler technology. URL: https://llvm.org/pubs/2008-05-17-BSDCan-LLVMIntro.html, 2008. Lecture at BSD Conference. 
[6] Michael, Zhivich and Robert, K. Cunningham. The real cost of software errors. IEEE Security \& Privacy, 7(2):87-90, 2009. DOI: 10.1109/MSP . 2009.56.

[7] Phabricator. Community conversion about loop widening. URL: https:// reviews.1lvm.org/D12358, 2015.

[8] Rice, Henry G. Classes of recursively enumerable sets and their decision problems. Trans. Amer. Math. Soc., 74:358-366, 1953. 